
conventional needles for lumbar puncture: a clinical practice guideline

\author{
Bram Rochwerg, ${ }^{12}$ Saleh A Almenawer, ${ }^{3}$ Reed A C Siemieniuk, ${ }^{2}{ }^{4}$ Per Olav Vandvik, 56 \\ Thomas Agoritsas, ${ }^{27}$ Lyubov Lytvyn, ${ }^{2}$ Waleed Alhazzani, ${ }^{12}$ Patrick Archambault,, 8910 \\ Frederick D'Aragon, ${ }^{11} 12$ Pauline Darbellay Farhoumand, ${ }^{7}$ Gordon Guyatt, ${ }^{2}$ Jon Henrik Laake, ${ }^{13}$ \\ Claudia Beltrán-Arroyave, ${ }^{14}$ Victoria McCredie, ${ }^{415}$ Amy Price, ${ }^{16}{ }^{17}$ Christian Chabot, $^{18}$ \\ Tracy Zervakis, ${ }^{19}$ Jetan Badhiwala, ${ }^{20}$ Maude St-Onge, ${ }^{10} 2122$ Wojciech Szczeklik, ${ }^{23}$ \\ Morten Hylander Møller, ${ }^{24} 25$ Francois Lamontagne ${ }^{11} 12$
}

\begin{abstract}
Full author details can be found at the end of the article

Correspondence to: FLamontagne francois.lamontagne@ usherbrooke.ca

Cite this as: BMJ 2018;361:k1920 doi: 10.1136/bmj.k1920
\end{abstract}

This BMJ Rapid Recommendation article is one of a series that provides clinicians with trustworthy recommendations for potentially practice changing evidence. BMJ Rapid Recommendations represent a collaborative effort between the MAGIC group (www. magicproject.org) and The $B M I$. A summary is offered here and the full version including decision aids is on the MAGICapp (www.magicapp.org), for all devices in multilayered formats. Those reading and using these recommendations should consider individual patient circumstances, and their values and preferences and may want to use consultation decision aids in MAGICapp to facilitate shared decision making with patients. We encourage adaptation and contextualisation of our recommendations to local or other contexts. Those considering use or adaptation of content may go to MAGICapp to link or extract its content or contact The BM/ for permission to reuse content in this article.
Is the needle tip configuration important when performing a lumbar puncture for any indication? A systematic review published in the Lancet in December 2017 suggests that it is. The review found that using atraumatic (pencil-point) lumbar puncture needles instead of conventional lumbar puncture needles reduced the risk of post-dural-puncture headache and of return to hospital for additional pain control. ${ }^{1}$ This guideline recommendation aims to promptly and transparently translate this evidence to a clinical recommendation, following standards for GRADE methodology and trustworthy guidelines. ${ }^{2}$ The BMJ Rapid Recommendations panel makes a strong recommendation for the use of atraumatic needles for lumbar puncture in all patients regardless of age (adults and children) or indication instead of conventional needles. ${ }^{34}$ Box 1 shows the article and evidence linked to this Rapid Recommendation. The main infographic provides an overview of the absolute benefits and harms (although none were present here) of atraumatic needles. Table 1 below shows any evidence that has emerged since the publication of this guideline.

Box 1 | Linked resources for this BMJRapid Recommendations cluster

- Rochwerg B, Almenawer SA, Siemieniuk RAC, et al. Atraumatic (pencil-point) versus conventional needles for lumbar puncture: a clinical practice guideline. $B M J$ 2018;361:k1920

Summary of the results from the Rapid Recommendation process

- Nath S, Koziarz A, Badhiwala JH, et al. Atraumatic versus conventional lumbar puncture needles: a systematic review and meta-analysis. Lancet 2018;391:1197-204 - Review of all available randomised trials comparing the use of atraumatic needles and conventional needles for any lumbar puncture indication

- MAGICapp (www.magicapp.org/public/guideline/j7A5Gn)

- Expanded version of the results from the Rapid Recommendation process with multilayered recommendations, evidence summaries, and decision aids for use on all devices

\section{WHAT YOU NEED TO KNOW}

- Post-dural-puncture headache is a common complication after lumbar puncture, affecting up to $35 \%$ of patients

- This headache results from sustained leakage of cerebrospinal fluid from a dural tear; it can be debilitating and require return to hospital for narcotics or invasive therapy

- We issue a strong recommendation for use of atraumatic needles in all patients (adults and children) undergoing lumbar puncture because they decrease complications and are no less likely to work than conventional needles

- Atraumatic needles are more expensive, but evidence suggests that they reduce costs overall compared with conventional needles

\section{Current practice}

Physicians perform lumbar punctures for diagnostic or therapeutic purposes. Among the complications associated with this procedure, post-dural-puncture headache is the most common, affecting up to $35 \%$ of patients. ${ }^{5}$ This complication can be debilitating, requiring return visits to the hospital for controlled analgesia, invasive therapy, or increased hospital duration of stay. ${ }^{56}$

Post-dural-puncture headache, among other adverse effects of lumbar punctures, is attributed to the leakage of cerebrospinal fluid from the dural defect into the epidural space that is created by the spinal needle during puncture.

Conventional needles have a sharp tip (designed to cut through tissues) and a distal opening. In comparison, atraumatic needles are more blunt with a closed pencilpoint or cone shaped tip and a side port for injection or collection.

Cadaveric studies using histological examination have shown that, compared with conventional needles, atraumatic needles more often separate and dilate surrounding dural fibres rather than cutting through them. Subsequent contracture of the fibres after needle removal results in a small pinpoint opening in the dura, as opposed to the 

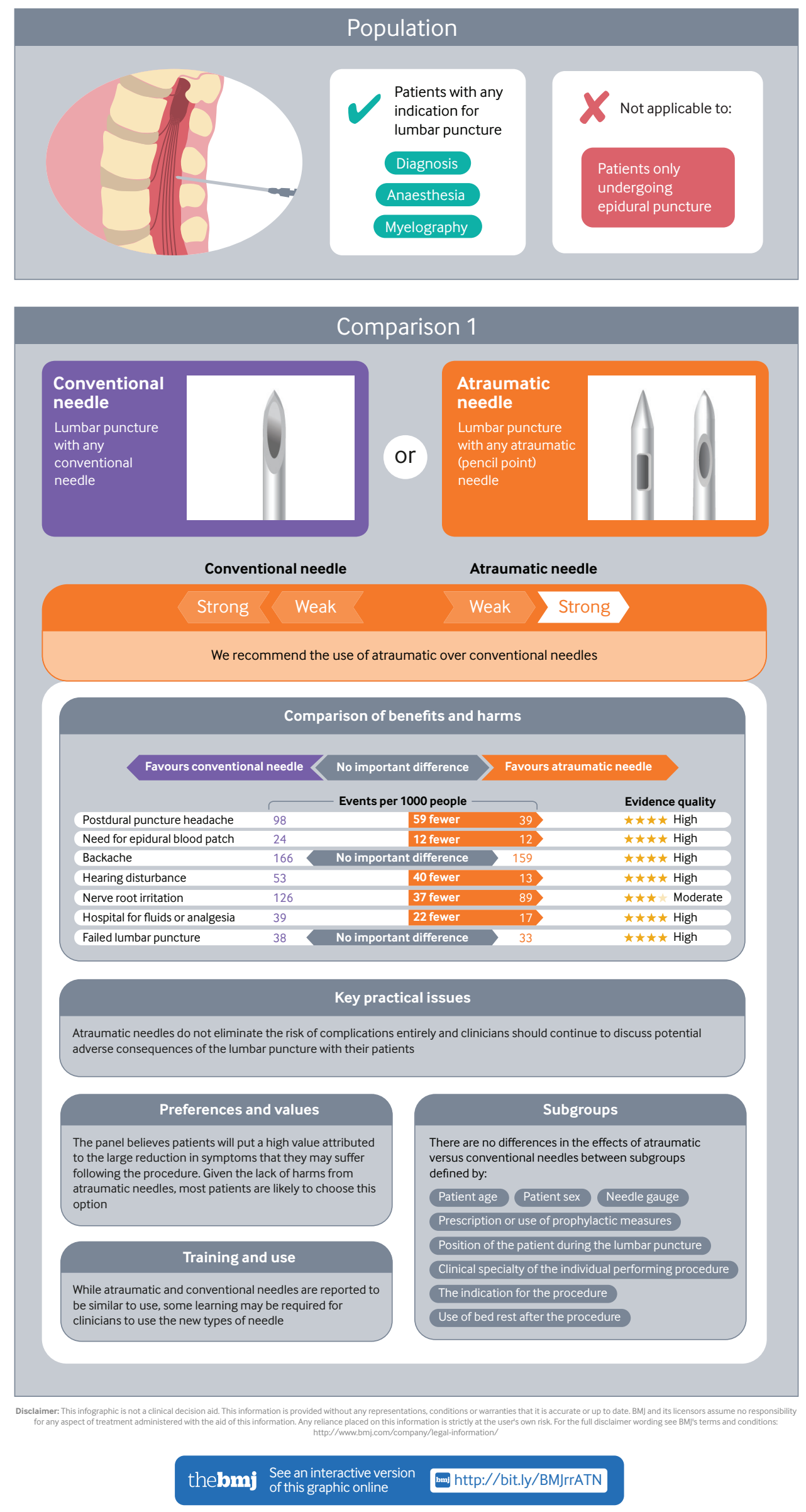


\section{DATA SOURCES similar your patients' conditions are}

to those of people studied in the trials

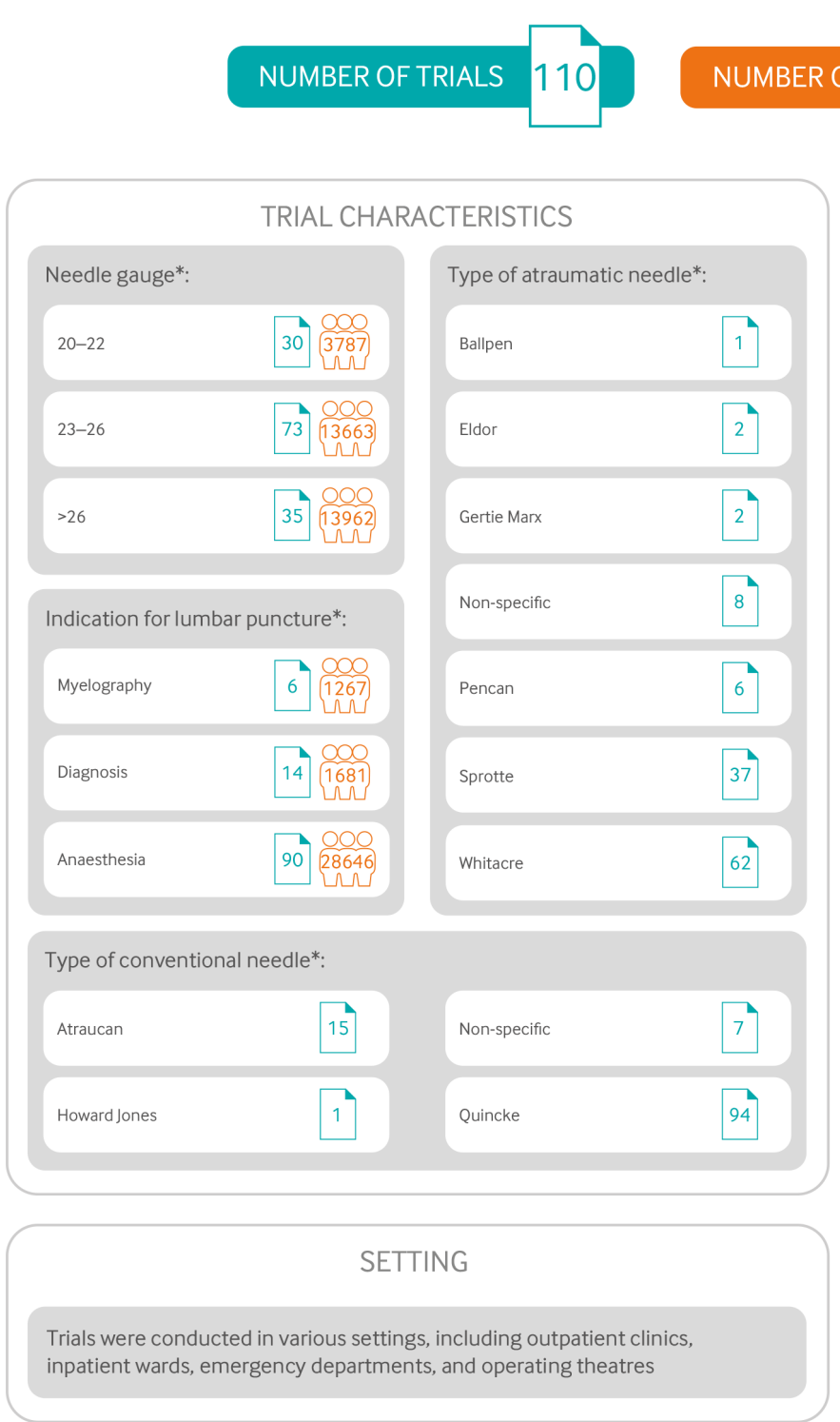

PATIENT CHARACTERISTICS

NUMBER OF

PATIENTS ENROLLED
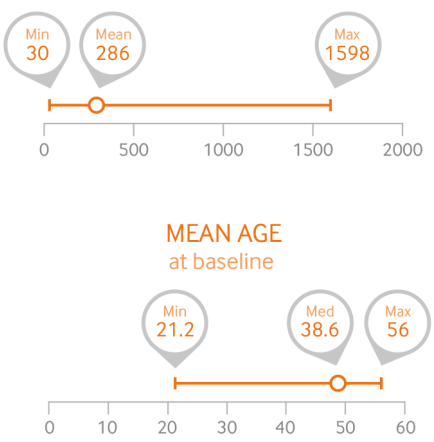

SEX
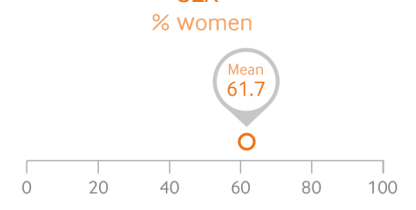

EXCLUSION CRITERIA

No dural puncture performed (epidural injection)

Study design (observational studies, reviews, commentaries, letters)

Studies comparing subtypes of atraumatic needles or subtypes of conventional

needles, without comparing atraumatic and conventional needles to one another

*Note number of trials do not add to 110 trial and percentages do not add to $100 \%$ as some trials examined more than one needle type, subtype, gauge, and indication.

Graphic 2 |Characteristics of patients and trials included in systematic review of the effects of needle type on the risk of major adverse effects associated with lumbar puncture

irregular and larger opening created by conventional needles. ${ }^{7}$ Use of atraumatic needles may therefore reduce the incidence of post-dural-puncture headache by limiting the leakage of cerebrospinal fluid after lumbar puncture. Surveys indicate that use of atraumatic needles in routine clinical practice is limited. ${ }^{8-10}$

Although terminology varies, for the purposes of this guideline, we will refer to atraumatic needles and conventional needles, which have a sharp tip to cut through tissues with a distal tip opening.

\section{The evidence}

The systematic review summarised the results of 110 randomised clinical trials (RCTs) conducted between 1989 and 2017 in 29 countries (including both high and middle/low income): it suggests that atraumatic needles consistently reduce the risk of major adverse effects associated with lumbar puncture done for any indication compared with conventional needles. More specifically, the risk of post-dural-puncture headache was significantly reduced when atraumatic needles were used for lumbar puncture (relative risk 0.40 (95\% confidence interval 0.34 to 0.47$)$ ). Graphic 2 presents an overview of the number and types of patients, as well as a summary of the benefits and harms (although none were present here) of atraumatic needles for lumbar punctures.

Individuals who were included in the eligible studies underwent lumbar punctures for any diagnostic or therapeutic indication. Baseline characteristics were similar between atraumatic and conventional needle groups, 


\section{HOW THIS RECOMMENDATION WAS CREATED}

The recent publication of a systematic review and meta-analysis of studies comparing atraumatic with conventional needles for any lumbar puncture triggered the following guideline recommendation. ${ }^{1}$ The Rapid Recommendations team believed that the results of this systematic review, which considered the full body of evidence, had important clinical implications and might change practice. ${ }^{2}$

Our international panel-including intensivists, neuro-intensivists, internists, anaesthesiologists, neurologists, neurosurgeons, emergency physicians, paediatricians, methodologists, and people with lived experience of lumbar puncture and caring for those with lumbar puncture-decided on the scope of the recommendation and the outcomes most important to patients (see appendix 1 on bmj.com). The panel met to discuss the evidence and formulate a recommendation. No panel member had financial conflicts of interest; intellectual and professional conflicts were minimised and transparently described (appendix 2 on bmj.com).

The panel followed the BMJ Rapid Recommendations procedures for creating a trustworthy recommendation, ${ }^{211}$ including using the GRADE approach to critically appraise the evidence and create recommendations (see appendix 3 on bmj.com). ${ }^{3}$ The panel considered the benefits, as well as any harms, and burdens of atraumatic needles, the certainty (quality) of the evidence for each outcome, typical and expected variations in patient values and preferences, acceptability, feasibility, and resource implications. ${ }^{12}$ Following the GRADE based approach, recommendations can be strong or weak (also known as conditional) for or against a specific course of action. ${ }^{13}$

The panel identified 13 patient-important outcomes to inform the recommendation: postdural-puncture headache (severe or mild), any headache (including those not meeting the exact definition of post-dural-puncture), backache, hearing disturbance, nerve root irritation, traumatic tap, need to return to hospital for intravenous fluids or controlled analgesia or for an epidural blood patch, failed lumbar puncture, successful lumbar puncture on first attempt, and cerebrospinal fluid drainage efficiency. There was disagreement among panel members whether to include this last outcome (drainage efficiency), but it was specifically prioritised by one of the patients. At least one of the included studies reported on all of these outcomes except for drainage efficiency, although the number of participants included in the analysis for each outcome varied. A few other patient-important outcomes such as mortality, brain herniation, quality of life, and permanent paralysis were considered, but their incidence after lumbar puncture was considered to be too rare to be informative to the recommendation.

with the exception of needle gauge, which was larger in the conventional needle group (larger gauge equals smaller needle diameter).

Only 1065 of the 31412 participants were children (3\%). The proportion of elderly participants was unknown. The results were consistent across the predefined subgroups including:

- Age $<18 v \geq 18$ years old

- Males $v$ females,

- Bed rest after lumbar puncture $v$ no bed rest

- Prophylactic intravenous fluids $v$ no prophylactic intravenous fluids

- Needle gauge (small $v$ large)

- Lateral $v$ sitting position during lumbar puncture

- Indication for lumbar puncture (anaesthesia $v$ diagnosis $v$ myelography)

- Clinical specialty of person doing the lumbar puncture (radiologist $v$ neurologist $v$ anaesthesiologist).

\section{Understanding the recommendation}

The guideline panel makes a strong recommendation for the use of atraumatic over conventional needles in lumbar puncture for any indication because the benefits are perceived to be large with no associated harm.

The panel is confident that the recommendation applies to all patients (adults and children) who require a lumbar puncture and all physicians as the results were consistent across all predefined subgroups mentioned above.
In addition the panel agreed that there is minimal variability in patient preferences to favour the use of atraumatic needles. ${ }^{1}$

\section{Absolute benefits and harms}

The main infographic explains the recommendation and provides an overview (GRADE summary of findings) of the absolute benefits of atraumatic needles. Estimates of baseline risk for effects are generated from the control arms of the included trials. ${ }^{1}$ The infographic also leads to point-of-care formats in the MAGICapp. ${ }^{14}$

The panel was confident that:

- Use of atraumatic needles meaningfully decreases the risk of postdural puncture headache (both severe and mild), any headache, hearing disturbance, nerve root irritation, return to hospital for intravenous fluids and controlled analgesia or need for epidural blood patch (GRADE high to moderate quality evidence)

- Use of atraumatic needles has little or no effect on the risk of backache (GRADE high quality evidence)

- Use of atraumatic needles has little or no effect on the incidence of traumatic tap, failed lumbar puncture, and probability of success on first attempt (GRADE high to moderate quality evidence)

- There are no differences in the effects of atraumatic versus conventional needles between subgroups defined by age and sex of patients, the prescription or use of prophylactic measures, needle gauge, position of the patient during the lumbar puncture, the clinical specialty of the individual performing the lumbar puncture, or the indication for the procedure

- It is unlikely that new information will change interpretation for outcomes for which the evidence is of high to moderate quality.

The panel was less confident about whether:

- Use of atraumatic needles affects the efficiency of cerebrospinal fluid drainage (that is, the time required to draw the necessary amount of cerebrospinal fluid) regardless of the indication. It is likely there are other more important factors that influence drainage efficiency than just needle type. Also, this outcome is of varying importance depending on the context and indication for lumbar puncture.

- The panel believed that the recommendation is generalisable even to patients who are unconscious, such as those who are mechanically ventilated and sedated in the intensive care unit as data suggests that post-dural-puncture headache can persist for several days and can be felt even under sedation. Increased pain in this population may lead to undesirable indirect effects such as increased heart rate and increased sedation or analgesic requirement.

\section{Values and preferences}

The panel placed high value on the large reduction in symptoms. The panel believes that values and preferences regarding all important outcomes are unlikely to vary greatly across patients, particularly given the lack of detectable harm from atraumatic needles. We do not 


\section{HOW PATIENTS WERE INVOLVED IN THE CREATION} OF THIS ARTICLE

Two people with lived experience of lumbar punctures, and one person with experience as a patient and a carer, were members of the guidance panel and authors. They identified and rated outcomes, and led the discussion on values and preferences. The patient partners rated all included outcomes as important to them. Although these values may not be shared by all patients for all outcomes considered, the panel expected little variation in how much importance other patients would place on the main outcomes of severe post-dural-puncture headache and the need to return to the hospital for an epidural blood patch. All panel members participated in the teleconferences and email discussions and met all authorship criteria.

anticipate that patients would opt for lumbar puncture needles associated with a greater risk of severe headaches. In contrast, the panel believes that there is considerable variability in how much importance individual patients and physicians attribute to traumatic taps (lumbar punctures contaminated with red blood cells negatively affecting fluid analysis). Accordingly, this outcome was considered to have limited importance in the recommendation.

The panel felt confident that atraumatic needles would be acceptable to patients, although this was not measured in the systematic review. Most clinicians found atraumatic and conventional needles similar to use. Some clinicians expressed potential concern regarding puncturing of the skin with the blunter atraumatic needle; however, this can be overcome by inserting the lumbar puncture needle through the same skin hole used for local anaesthesia, by using an introducer needle, or by spinning the atraumatic needle around its axis while advancing the needle. ${ }^{1}$

\section{Practical issues and other considerations}

Atraumatic needles do not eliminate the risk of complications entirely, and clinicians should continue to discuss potential adverse consequences of the lumbar puncture with their patients.

\section{Costs and resources}

The panel reviewed three published cost-effectiveness studies. ${ }^{15-17}$ In those studies, the per-unit cost of atraumatic needles was greater than the cost of conventional needles, but atraumatic needles were ultimately costreducing because of the decreased need for additional care (perspective of the third-party payer) and lost working hours for patients (perspective of the patients and society). Moreover, as with conventional needles, the per-unit cost varies with the specific needle subtype and manufacturer.

\section{Uncertainties}

Addressing the following remaining knowledge gaps may inform decision makers and future guideline recommendations:

- Given the plausible greater risk of spinal stenosis and degenerative process in elderly patients who require a lumbar puncture, is the success rate for atraumatic and conventional needles similar in this population?

\begin{tabular}{l} 
New evidence which has emerged after initial publication \\
$\qquad \begin{array}{l}\text { New } \\
\text { Date evidence } \quad \text { Implications for } \\
\text { There are currently no updates to the article. }\end{array}$ \\
Thecommendation(s) \\
\hline
\end{tabular}

\section{EDUCATION INTO PRACTICE}

- When performing a lumbar puncture, which needles do you use? Why?

- Based on this article how do you think your personal practice might change? Is there anything that you would say to a patient or do differently?

- How might you share this information with your organisation or review local policies on needle choice?

- Are certain subtypes of atraumatic needles (such as Cappe-Deutsch, Eldor, Gertie-Marx, Microtip, Sprotte, or Whitacre) associated with greater reduction in adverse events than others?

\section{Updates to this article}

The table shows evidence which has emerged since the publication of this article. As new evidence is published, a group will assess the new evidence and make a judgment on to what extent it is expected to alter the recommendation.

Competing interests: All authors have completed the BMJ Rapid Recommendations interests disclosure form, and a detailed description of all disclosures is reported in appendix 2 on bmi.com. As with all BMJ Rapid Recommendations, the executive team and The BM/judged that no panel member had any financial conflict of interest. Professional and academic interests are minimised as much as possible, while maintaining necessary expertise on the panel to make fully informed decisions. Three authors of the systematic review were on the guideline panel (WA, SAA, JB). Funding: This guideline was not funded.

Transparency: B Rochwerg affirms that the manuscript is an honest, accurate, and transparent account of the recommendation being reported; that no important aspects of the recommendation have been omitted; and that any discrepancies from the recommendation as planned (and, if relevant, registered) have been explained.

1 Nath S, Koziarz A, Badhiwala JH, et al. Atraumatic versus conventional lumbar puncture needles: a systematic review and meta-analysis. Lancet 2018;391:1197-204. 10.1016/S0140-6736(17)324510 pmid:29223694.

2 Siemieniuk RA, Agoritsas T, Macdonald H, Guyatt GH, Brandt L, Vandvik PO Introduction to BMJ Rapid Recommendations. BMJ 2016;354:15191. 10.1136/bmj.i5191 pmid:27680768

3 Guyatt GH, Oxman AD, Vist GE, et al. GRADE Working Group. GRADE: an emerging consensus on rating quality of evidence and strength of recommendations. BM/ 2008;336:924-6. 10.1136/ bmj.39489.470347.AD pmid:18436948.

4 Institute of Medicine (US) Committee on Standards for Developing Trustworthy Clinical Practice Guidelines; eds. Graham R, Mancher M, Miller Wolman D, et al. Clinical practice guidelines we can trust. National Academies Press, 2011. https://www.ncbi.nlm.nih.gov/books/ NBK209539/.

5 Lavi R, Yarnitsky D, Rowe JM, Weissman A, Segal D, Avivi I. Standard vs atraumatic Whitacre needle for diagnostic lumbar puncture: a randomized trial. Neurology 2006;67:1492-4. 10.1212/01. wnl.0000240054.40274.8a pmid:17060584.

6 Turnbull DK, Shepherd DB. Post-dural puncture headache: pathogenesis, prevention and treatment. BrJ Anaesth 2003;91:718-29. 10.1093/bja/ aeg231 pmid:14570796

7 Celleno D, Capogna G, Costantino P, Catalano P. An anatomic study of the effects of dural puncture with different spinal needles. Reg Anesth 1993:18:218-21.pmid:8398954.

8 Birnbach DJ, Kuroda MM, Sternman D, Thys DM. Use of atraumatic spinal needles among neurologists in the United States. Headache 2001;41:385-90. 10.1046/j.15264610.2001.111006385.x pmid:11318885.

9 Davis A, Dobson R, Kaninia S, Giovannoni G, Schmierer K. Atraumatic needles for lumbar puncture: why haven't neurologists changed?Pract Neurol 2016;16:18-22. 10.1136/ practneurol-2014-001055 pmid:26349834. 
10 Moisset X, Ruet A, Brochet B, et al. Who performs lumbar puncture, how many do they perform, how and why? A retrospective study of 6,594 cases. Eur Neurol 2016:76:8-11. 10.1159/000447452 pmid:27336667.

11 Vandvik PO, Otto CM, Siemieniuk RA, et al. Transcatheter or surgical aortic valve replacement for patients with severe, symptomatic, aortic stenosis at low to intermediate surgical risk: a clinical practice guideline. BM 2016;354:i5085. 10.1136/bmj.i5085 pmid:27680583.

12 Andrews IC Schünemann HI Oxman AD et al GRADE guidelines: 15. Going from evidence to recommendation-determinants of a recommendation's direction and strength. J Clin Epidemiol 2013;66:72635. 10.1016/j.jclinepi.2013.02.003 pmid:23570745.

13 Guyatt GH, Oxman AD, Kunz R, et al. GRADE Working Group. Going from evidence to recommendations. BMI 2008:336:1049-51.10.1136 bmj.39493.646875.AE pmid:18467413.

14 Agoritsas T, Heen AF, Brandt L, et al. Decision aids that really promote shared decision making: the pace quickens. BMJ 2015;350:97624 10.1136/bmj.g7624 pmid:25670178.

15 Dakka Y, Warra N, Albadareen RJ, Jankowski M, Silver B. Headache rate and cost of care following lumbar puncture at a single tertiary care hospital. Neurology 2011;77:71-4.10.1212/ WNL.0b013e318220abc0 pmid:21593442.

16 Engedal TS, Ørding H, Vilholm OJ. Changing the needle for lumbar punctures: results from a prospective study. Clin Neurol Neurosurg 2015;130:74-9.10.1016/i.clineuro.2014.12.020 pmid:25590665.

17 Tung CE, So YT, Lansberg MG. Cost comparison between the atraumatic and cutting lumbar puncture needles. Neurology 2012;78:109-13. 10.1212/WNL.0b013e31823efca9 pmid:22205758.

Published by the BMJ Publishing Group Limited. For permission to use (where not already granted under a licence) please go to http://group. bmj.com/group/rights-licensing/permissions

This is an Open Access article distributed in accordance with the Creative Commons Attribution Non Commercial (CC BY-NC 4.0) license, which permits others to distribute, remix, adapt, build upon this work non-commercially, and license their derivative works on different terms, provided the original work is properly cited and the use is noncommercial. See: http://creativecommons.org/licenses/by-nc/4.0/
${ }^{1}$ Department of Medicine, McMaster University, Hamilton, Ontario, Canada ${ }^{2}$ Department of Health Research Methods, Evidence and Impact, McMaster University, Hamilton, Canada

${ }^{3}$ Division of Neurosurgery, McMaster University, Hamilton, Canada ${ }^{4}$ Department of Medicine, University of Toronto, Toronto, Ontario, Canada ${ }^{5}$ Institute of Health and Society, Faculty of Medicine, University of Oslo, Oslo, Norway

${ }^{6}$ Department of Medicine, Innlandet Hospital Trust-division, Gjøvik, Norway ${ }^{7}$ Division General Internal Medicine \& Division of Clinical Epidemiology, University Hospitals of Geneva, Geneva, Switzerland

${ }^{8}$ Department of Family Medicine and Emergency Medicine \& Department of Anesthesiology and Critical Care Medicine, Université Laval, Québec City, Canada

${ }^{9}$ Centre intégré de santé et de services sociaux de Chaudière-Appalaches, Centre hospitalier affilié universitaire Hôtel-Dieu de Lévis, Lévis, Canada

${ }^{10} \mathrm{CHU}$ de Québec - Université Laval Research Center, CHU de Québec -

Université Laval, Université Laval, Québec City, Canada

${ }^{11}$ Faculty of Medicine and Health Sciences Université de Sherbrooke,

Sherbrooke, Canada

${ }^{12}$ Research Centre, Centre Hospitalier Universitaire de Sherbrooke,

Sherbrooke, Canada

${ }^{13}$ Department of Anaesthesiology, Division of Emergency and Critical Care, Rikshospitalet Medical Centre, Oslo University Hospital, Oslo, Norway

${ }^{14}$ Department of Pediatrics, Universidad de Antioquia, Medellin, Colombia

${ }^{15}$ Division of Critical Care Medicine, Department of Medicine, University Health Network, Toronto, Canada

${ }^{16}$ The BMJ (Research and Evaluation), London, UK

${ }^{17}$ Department of Continuing Education, University of Oxford, Oxford, UK

${ }^{18}$ McGill University, Montreal, Canada

${ }^{19}$ Virginia, USA

${ }^{20}$ Department of Surgery, University of Toronto, Toronto, Canada

${ }^{21}$ Centre intégré de santé et de services sociaux de la Capitale-Nationale, Québec City, Canada

${ }^{22}$ Department of Family and Emergency Medicine \& Department of Anesthesiology and Critical Care \& Faculty of Medicine, Université Laval, Laval, Canada

${ }^{23}$ Department of Intensive Care and Perioperative Medicine, Jagiellonian University Medical College, Kraków, Poland

${ }^{24}$ Department of Intensive Care, Copenhagen University Hospital Rigshospitalet, Copenhagen, Denmark

${ }^{25}$ Centre for Research in Intensive Care (CRIC), Copenhagen, Denmark 